\title{
Constrained Minimax Optimization of Continuous Search Efforts for the Detection of a Stationary Target
}

\author{
Frédéric Dambreville, ${ }^{1}$ Jean-Pierre Le Cadre ${ }^{2}$ \\ ${ }^{1}$ Délégation Générale pour l'Armement, 16 Bis, Avenue Prieur de la Côte d'Or, 94114 Arcueil, France \\ ${ }^{2}$ IRISA/CNRS, Campus de Beaulieu, 35042 Rennes Cedex, France
}

Received 29 September 2006; revised 30 January 2007; accepted 30 January 2007

DOI 10.1002/nav.20229

Published online 28 March 2007 in Wiley InterScience (www.interscience.wiley.com).

\begin{abstract}
Analytical resolution of search theory problems, as formalized by B.O. Koopman, may be applied with some model extension to various resource management issues. However, a fundamental prerequisite is the knowledge of the prior target density. Though this assumption has the definite advantage of simplicity, its drawback is clearly that target reactivity is not taken into account. As a preliminary step towards reactive target study stands the problem of resource planning under a min-max game context. This paper is related to Nakai's work about the game planning of resources for the detection of a stationary target. However, this initial problem is extended by adding new and more general constraints, allowing a more realistic modeling of the target and searcher behaviors. () 2007 Wiley Periodicals, Inc. Naval Research Logistics 54: 589-601, 2007
\end{abstract}

Keywords: sensor and resource management; target detection; search game; search theory; resource allocation

\section{NOTATIONS}

$\varphi(x)$ : Search effort

$\phi_{o}:$ Total amount of search effort

$\alpha(x)$ : Probabilistic target distribution

$A_{o}$ : Probability of the target to be in the search area $p_{x}(\varphi(x))$ : Conditional nondetection probability

\section{INTRODUCTION}

The initial framework of Search Theory $[1,8,14,16]$, introduced by B.O. Koopman and his colleagues, sets the general problem of the detection of a target in a space, in view of optimizing the use of the detection resources. A thorough extension of the prior formalism has been made by Brown towards the detection at several periods of search $[2,17]$. This simple but meaningful formalism has also been applied to various resource management involving data fusion issues [3]. But, for all these problems, a probabilistic prior on the target was required. In addition, in case of moving target problems, a Markovian hypothesis is necessary for algorithmic reasons. While this formalism is sufficient for almost

Correspondence to: F. Dambreville (science_publications@ fredericdambreville.com) "passive" targets, it may be dubious and even useless when a target has a complex (and realistic) move. In a military context especially, the behavior of the "interesting" targets is not neutral and cannot be modeled by a simple probabilistic prior. A conceivable way for improving the prior about the target in a manner that involves more properly the complexity or the reactive behavior of the target, is to consider a min-max game version of the Koopman optimization problems. Nakai presented and solved in [9] a game version of the resource planning for the detection of a stationary target. In this work the constraints on game were given by the available location of the target on the one hand and the amount of detection resources on the other. Thus, the constraints were defined at the pure strategy level. The purpose of this paper is to present an extension of Nakai's game by addition of new constraints defined on the set of available mixed strategies. In other words, constraints are now defined at the mixed strategy level. Before explaining properly the extended problem, we intend to give in this introduction a short description of Nakai's game.

Definitions. The searcher wants to detect a target located in a search space $E$. To perform this detection, the searcher has available a total amount of (detection) resources $\phi_{o}$. These resources may be put on each cell $x$ of the search space $E$. Detection on cell $x$ is a known function of the search effort 
put on $x$. For $x \in E$, the variable $\varphi(x)$ denotes the local amount of resources placed on cell $x$. A constraint naturally holds for the global amount of resources in use:

$$
\sum_{x \in E} \varphi(x) \leq \phi_{o}
$$

Since the detection is maximized when the whole resources are used, the previous constraint may be replaced by an equality one:

$$
\sum_{x \in E} \varphi(x)=\phi_{o}
$$

The set of valid sharing functions $\varphi$ is thus defined by

$$
\mathcal{R}(\phi)=\left\{\varphi \in \mathbb{R}^{+E} / \sum_{x \in E} \varphi(x)=\phi_{o}\right\} .
$$

When the local resource $\varphi(x)$ is put on cell $x$ and the target is located on $x$, the conditional probability of nondetection is given by the value of $p_{x}(\varphi(x))$. This probability may depend upon $x$, since visibility and resource efficiency vary practically with the concerned cell. For $x$ fixed, it is usually assumed that $p_{x}(0)=1$ (no search implies no detection) and $p_{x}$ decreases with the effort used, i.e. $p_{x}^{\prime}<0$. It is also hypothesized that the detection follows the rule of decreasing return, so that $p_{x}^{\prime}$ increases strictly with $\varphi$. On the other hand, the target have the choice between several available locations, described by the subset $\mathbb{T} \subset E$. Then, a game occurs between the searcher and the target. The searcher attempts to minimize the probability of nondetection by optimizing the search resource sharing $\varphi$, while the target's aim is to maximize the probability of nondetection by choosing his location. The evaluation of the game is given by $p_{x}(\varphi(x))$, for a pure target strategy $x$ and a pure searcher strategy $\varphi$. A mixed strategy for the target is given by a density probability $\alpha$ on the target location, with property $\alpha(E \backslash \mathbb{T})=0$. We denote

$$
\mathcal{P}(\mathbb{T})=\left\{\alpha \in \mathbb{R}^{+E} / \alpha(E \backslash \mathbb{T})=0 \text { and } \sum_{x \in E} \alpha(x)=1\right\},
$$

the set of such probabilities. For a strategy pair $(\alpha, \varphi)$, the evaluation of the (minimization) game is then given by the averaged value (denoted $P_{\text {nd }}$ ) of the probability of nondetection.

$$
P_{n d}(\alpha, \varphi)=\sum_{x \in E} \alpha(x) p_{x}(\varphi(x))
$$

This game was solved by Nakai [9]. Since $p$ is convex, it appears that the game is convex. Thus, there is a mixed optimal strategy for the target and a pure optimal strategy for the searcher. A saddle point strategy $\left(\alpha_{o}, \varphi_{o}\right)$ is defined by

$$
\left\{\begin{array}{l}
\alpha_{o}=\arg \max _{\alpha \in \mathcal{P}(\mathbb{T})} \sum_{x \in E} \alpha(x) p_{x}\left(\varphi_{o}(x)\right), \\
\varphi_{o}=\arg \min _{\varphi \in \mathcal{R}\left(\phi_{o}\right)} \sum_{x \in E} \alpha_{o}(x) p_{x}(\varphi(x)) .
\end{array}\right.
$$

Two optimality conditions are obtained, by differentiation around the optimal strategies:

$$
\exists \eta<0, \begin{cases}\alpha_{o}(x) p_{x}^{\prime}\left(\varphi_{o}(x)\right)=\eta, & \text { when } \alpha_{o}(x)>\frac{\eta}{p_{x}^{\prime}(0)} \\ \varphi_{o}(x)=0, & \text { else }\end{cases}
$$

and

$$
\exists \lambda \geq 0, \alpha_{o}(x)>0 \Longrightarrow p_{x}\left(\varphi_{o}(x)\right)=\lambda .
$$

In Eq. (2) is recognized the classical optimality equation of de Guenin [4]. By use of these equations, a mathematical solution of the problem is built. The first step is to verify the obviously intuitive result:

PROPOSITION 1: Let $\mathbb{T}$ be the space of possible target locations; then the following equivalence holds true:

$$
x \in \mathbb{T} \Longleftrightarrow \alpha_{o}(x)>0 \Longleftrightarrow \varphi_{o}(x)>0 .
$$

PROOF OF PROPOSITION 1: If there is a cell, say $a \in \mathbb{T}$, such that $\varphi_{o}(a)=0$, then $p_{a}\left(\varphi_{o}(a)\right)=1$ and the pure target strategy $a$ yields the maximal evaluation, 1 , for the game. This contradicts the hypothesis that $\varphi_{o}$ is a min-max strategy. It results that $x \in \mathbb{T} \Longrightarrow \varphi_{o}(x)>0$.

Assume now $\varphi_{o}(x)>0$. From Eq. (2), then holds $\alpha_{o}(x)>$ $\frac{\eta}{p_{x}^{\prime}(0)}$. Thus, $\varphi_{o}(x)>0 \Longrightarrow \alpha_{o}(x)>0$.

The implication $\alpha_{o}(x)>0 \Longrightarrow x \in \mathbb{T}$ is obvious from the definition of $\mathcal{P}(\mathbb{T})$.

Notice particularly that this proof requires the values $p_{x}(0)$ be equal to 1 . But the property still holds when $p_{x}(0)$ is independent of $x$.

Now, the combination of Eq. (1) and (3) yields $\sum_{x \in \mathbb{T}} p_{x}^{-1}(\lambda)=\phi_{o}$. Defining the function $\mathbb{P}$ by

$$
\mathbb{P}^{-1}(\lambda)=\sum_{x \in \mathbb{T}} p_{x}^{-1}(\lambda)
$$

it follows that $\lambda=\mathbb{P}\left(\phi_{o}\right)$. Finally, the Eqs. (2) and (3) simplify and reduce to $\varphi_{o}(x)=p_{x}^{-1}\left(\mathbb{P}\left(\phi_{o}\right)\right)$ and $\alpha_{o}(x)=$ $\eta / p_{x}^{\prime}\left(p_{x}^{-1}\left(\mathbb{P}\left(\phi_{o}\right)\right)\right)$. Since $\alpha_{o}$ is a probability density, it follows that $\sum_{x \in \mathbb{T}} \alpha_{o}(x)=1$. The dual variable $\eta$ is then deduced. After simplification, the simple formula $\eta=\mathbb{P}^{\prime}\left(\phi_{o}\right)$ 
is obtained. The saddle point strategy $\left(\alpha_{o}, \varphi_{o}\right)$ is simply given by

$$
\forall x \in \mathbb{T},\left\{\begin{array}{l}
\alpha_{o}(x)=\left(p_{x}^{-1} \circ \mathbb{P}\right)^{\prime}\left(\phi_{o}\right) \\
\varphi_{o}(x)=\left(p_{x}^{-1} \circ \mathbb{P}\right)\left(\phi_{o}\right)
\end{array}\right.
$$

Nakai's game thus admits a mathematical solution. It is interesting to compare it with the classical one-sided search problem of de Guenin [4], where $\alpha(x)$ is now a fixed prior and only $\varphi$ is optimized, i.e. $\varphi_{o}=$ $\arg \min _{\varphi \in \mathcal{R}\left(\phi_{o}\right)} \sum_{x \in E} \alpha(x) p_{x}(\varphi(x))$. It happens that the searcher's strategy is less complex in the case of the game.

In the next section, an extension of Nakai's game will be considered. It is a zero sum game, wherein constraints are put on the target mixed strategies. Such problem will be seen as a generalization of both Nakai's game and de Guenin's problem, but is much more complex than these two parent problems. Particularly, there is no general mathematical solution. New properties will be established to handle these difficulties and an original algorithm will be presented.

\section{BOUNDING CONSTRAINTS}

In Nakai's game, the prior on target, $\mathbb{T}$, is given by the set of available target locations. This prior is more general and more flexible than a probabilistic density on the target location, in particular for modeling uncertain targets. Nevertheless, it does not allow sufficient refinement, for modeling the target behavior. For example, when the detection occurs after a preliminary target move, it is wise to handle the target motion modelling. Itself depending on the target reaction capabilities, it follows that some final locations are more probable than others. To model this fact, we will simply introduce lower and upper bounds on the probability associated with the target mixed strategy.

Similarly, operational considerations plead for introducing lower and upper bounding on the resources sharing functions. Doing that involves a symmetrization of our problem. However, such bounding constraints on resources have a physical meaning. It implies a minimum value and a maximum value of resource on each cell of the space search. Definitions have now to be clarified.

Definition. Again, the search of the target is made within a space $E$. Each element $x \in E$ is called a cell. A mixed strategy of the target is represented by a density function $\alpha$ defined on $E$. The function $\alpha$ is an unknown variable of the problem. The summation of $\alpha$ on $E$ is known and is denoted $A_{o}$. The following constraint then holds:

$$
\sum_{x \in E} \alpha(x)=A_{o}
$$

Since $\alpha$ is a density probability, $A_{o}$ generally equals 1 . But $A_{o}<0$ means that the target is possibly outside the search space. Two functions $\alpha_{1}$ and $\alpha_{2}$, mapping from $E$ to $\mathbb{R}^{+}$, are given with the property $0 \leq \alpha_{1} \leq \alpha_{2}$. These functions yield a constraint on the mixed target strategy:

$$
\alpha_{1} \leq \alpha \leq \alpha_{2} .
$$

A pure strategy of the searcher is represented by a resource sharing function $\varphi$ defined on $E$. The function $\varphi$ is also an unknown variable of the problem. The total amount of resources $\phi_{o}$ is fixed, so that

$$
\sum_{x \in E} \varphi(x)=\phi_{o}
$$

Two functions $\varphi_{1}$ and $\varphi_{2}$, mapping from $E$ to $\mathbb{R}^{+}$, are given with the property $0 \leq \varphi_{1} \leq \varphi_{2}$. These functions yield a constraint on the pure search strategy:

$$
\varphi_{1} \leq \varphi \leq \varphi_{2}
$$

For each cell $x$, the probability of nondetection is a decreasing and convex function $p_{x}$. The game evaluation of a strategy pair $(\alpha, \varphi)$ is given by the averaged probability of nondetection:

$$
P_{n d}(\alpha, \varphi)=\sum_{x \in E} \alpha(x) p_{x}(\varphi(x)) .
$$

Since the game is convex with respect to $\varphi$, there is a couple of optimal strategies involving a mixed strategy for the target and a pure strategy for the searcher. The associated zero sum game stands as follows:

Find $\alpha_{o}, \varphi_{o}$ such that

$$
\alpha_{o} \in \arg \max _{\alpha} \sum_{x \in E} \alpha(x) p_{x}\left(\varphi_{o}(x)\right)
$$

and

$$
\varphi_{o} \in \arg \min _{\varphi} \sum_{x \in E} \alpha_{o}(x) p_{x}(\varphi(x)),
$$

under constraints

$$
\begin{aligned}
& \forall x \in E, \alpha_{1}(x) \leq \alpha(x) \leq \alpha_{2}(x), \text { and } \sum_{x \in E} \alpha(x)=A_{o}, \\
& \forall x \in E, \varphi_{1}(x) \leq \varphi(x) \leq \varphi_{2}(x), \text { and } \sum_{x \in E} \varphi(x)=\phi_{o} .
\end{aligned}
$$

Summary of the problem setting.

- $\alpha_{1}$ : lower bound for the target mixed strategy

- $\alpha_{2}$ : upper bound for the target mixed strategy

- $\varphi_{1}$ : lower bound for the searcher strategy

- $\varphi_{2}$ : upper bound for the searcher strategy

- Constraints on the target mixed strategy:

$-\alpha_{1} \leq \alpha \leq \alpha_{2}$

$-\sum_{x \in E} \alpha(x)=A_{o}$ 
- Constraints on the searcher strategy:

$-\varphi_{1} \leq \varphi \leq \varphi_{2}$

$-\sum_{x \in E} \varphi(x)=\phi_{o}$

- $p_{x}(\varphi(x))$ : Conditional probability of nondetection, when resource $\varphi(x)$ is applied on cell $x$

$-p_{x}>0$

$-p_{x}^{\prime}(\varphi(x))<0$

$-p_{x}^{\prime \prime}>0$

Additional properties are hypothesized in order to ensure the existence of solutions:

$$
\begin{aligned}
\sum_{x \in E} \alpha_{1}(x) \leq A_{o} \leq \sum_{x \in E} \alpha_{2}(x) \text { and } \sum_{x \in E} \varphi_{1}(x) & \\
& \leq \phi_{o} \leq \sum_{x \in E} \varphi_{2}(x)
\end{aligned}
$$

\section{OPTIMALITY CONDITIONS}

Problem setting having been defined, we are now dealing with the derivation of the optimality conditions which constitute the core of this paper and are fundamental for deriving a practical optimization algorithm.

\subsection{Deriving the Optimality Conditions}

Considering a saddle point strategy $\left(\alpha_{o}, \varphi_{o}\right)$ for the game, two optimality equations are obtained by variational means.

de Guenin's condition. Since $\left(\alpha_{o}, \varphi_{o}\right)$ is a couple of saddle points, it appears that

$$
\varphi_{o} \in \arg \min _{\varphi} P_{n d}\left(\alpha_{o}, \varphi\right)
$$

The constraints $\varphi_{1} \leq \varphi \leq \varphi_{2}$ apply to the minimization. A result very similar to the condition of de Guenin is thus obtained, e.g. by applying the Karush-Kuhn-Tucker theorem. The following conditions hold true:

$\exists \eta<0$,

$$
\begin{cases}\varphi_{o}(x)=\varphi_{1}(x) & \text { if } \quad \varphi_{1}(x) \geq p_{x}^{\prime-1}\left(\eta / \alpha_{o}(x)\right), \\ \varphi_{o}(x)=\varphi_{2}(x) & \text { if } \quad \varphi_{2}(x) \leq p_{x}^{\prime-1}\left(\eta / \alpha_{o}(x)\right), \\ \varphi_{o}(x)=p_{x}^{\prime-1}\left(\eta / \alpha_{o}(x)\right) & \text { else. }\end{cases}
$$

Constancy condition. This condition is recovered from the maximization, applying for any saddle point:

$$
\alpha_{o} \in \arg \max _{\alpha} P_{n d}\left(\alpha, \varphi_{o}\right)
$$

Naval Research Logistics DOI 10.1002/nav
The constraints $\alpha_{1} \leq \alpha \leq \alpha_{2}$ apply to the maximization. By use of the KKT theorem, and since $\varphi \mapsto p_{x}(\varphi)$ is decreasing, the following conditions are refined:

$\exists \lambda \geq 0, \quad\left\{\begin{array}{llll}\varphi_{o}(x) \leq p_{x}^{-1}(\lambda) & \text { if } & \alpha(x)=\alpha_{2}(x)>\alpha_{1}(x), \\ \varphi_{o}(x) \geq p_{x}^{-1}(\lambda) & \text { if } & \alpha(x)=\alpha_{1}(x)<\alpha_{2}(x), \\ \varphi_{o}(x)=p_{x}^{-1}(\lambda) & \text { if } & \alpha_{1}(x)<\alpha(x)<\alpha_{2}(x) .\end{array}\right.$

Related curves. The previous conditions (6) and (7) have an obvious geometric interpretation. For a given cell $x$, the optimal strategies $\left(\alpha_{o}, \varphi_{o}\right)$ are locally defined by the intersection of two parameterized curves $H_{\eta}^{x}$ and $\Lambda_{\lambda}^{x}(\eta<0$ and $\lambda \geq 0)$. In other words, $\left(\alpha_{o}(x), \varphi_{o}(x)\right) \in H_{\eta}^{x} \cap \Lambda_{\lambda}^{x}$. These two curves are defined respectively from (6) and (7):

$(a, f) \in H_{\eta}^{x} \Leftrightarrow\left\{\begin{array}{l}a \leq \frac{\eta}{p_{x}^{\prime}\left(\varphi_{1}(x)\right)} \Rightarrow f=\varphi_{1}(x) \\ \frac{\eta}{p_{x}^{\prime}\left(\varphi_{1}(x)\right)}<a<\frac{\eta}{p_{x}^{\prime}\left(\varphi_{2}(x)\right)} \Rightarrow a p_{x}^{\prime}(f)=\eta \\ a \geq \frac{\eta}{p_{x}^{\prime}\left(\varphi_{2}(x)\right)} \Rightarrow f=\varphi_{2}(x)\end{array}\right.$

and

$$
(a, f) \in \Lambda_{\lambda}^{x} \Leftrightarrow\left\{\begin{array}{l}
f<p_{x}^{-1}(\lambda) \Rightarrow a=\alpha_{2}(x) \\
f=p_{x}^{-1}(\lambda) \Rightarrow a \in\left[\alpha_{1}(x), \alpha_{2}(x)\right] \\
f>p_{x}^{-1}(\lambda) \Rightarrow a=\alpha_{1}(x)
\end{array}\right.
$$

In this definition, the coordinates $a$ and $f$ are related to the variables $\alpha(x)$ and $\varphi(x)$. The conditions (6) and (7) have a more precise meaning. There is a common choice of dual variables, which defines the whole optimal strategies as local intersections of the associated curves.

$$
\exists \eta_{o}, \exists \lambda_{o}, \forall x \in E,\left(\alpha_{o}(x), \varphi_{o}(x)\right) \in H_{\eta_{o}}^{x} \cap \Lambda_{\lambda_{o}}^{x} .
$$

This point will be instrumental for developing a feasible algorithm.

Graphical meaning. Since $p_{x}$ is convex, it follows that $H_{\eta_{o}}^{x}$ is a nondecreasing curve. More precisely, $H_{\eta_{o}}^{x}$ is increasing for $\frac{\eta}{p_{x}^{\prime}\left(\varphi_{1}(x)\right)}<a<\frac{\eta}{p_{x}^{\prime}\left(\varphi_{2}(x)\right)}$ and flat otherwise. On the contrary, $\Lambda_{\lambda_{0}}^{x}$ is a nonincreasing curve, which is flat for $a \in] \alpha_{1}(x), \alpha_{2}(x)$ [ and infinitely decreasing otherwise. These two curves are sketched in Fig. 1. If these curves were strictly increasing/decreasing, there would have been only one intersection for $H_{\eta}^{x}$ and $\Lambda_{\lambda}^{x}$. But owing to the possible flatness of the curves, the intersection may be a segment, as shown in the left picture of Fig. 1. The nonuniqueness of the intersection is related to possibly undetermined cases for the optimality conditions. Thus, after tuning the dual parameters $\lambda, \eta$ in accordance with the resource constraints $\sum_{x \in E} \alpha(x)=A_{o}$ and $\sum_{x \in E} \varphi(x)=\phi_{o}$, it is still possible that several solutions are optimal. 

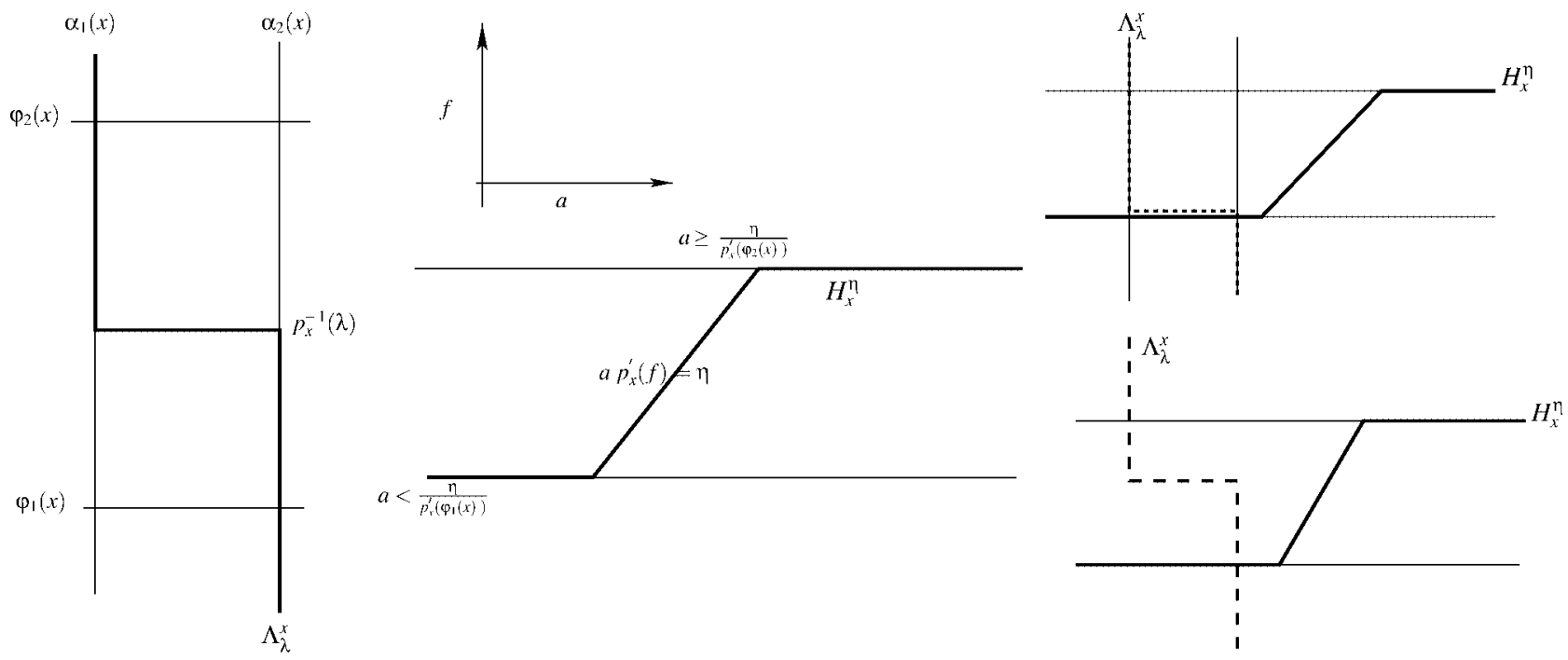

Figure 1. Curves $\Lambda_{\lambda}^{x}$ and $H_{\eta}^{x}$ : defined and undefined intersection cases.

\subsection{Inverting the Optimality Conditions}

Optimality conditions have been derived in Section 3.1. In this section, the inversion of these conditions is investigated.

Dual mapping. For a given choice $(\eta, \lambda)$ of the dual variables, the set of possible saddle points is $H_{\eta}^{x} \cap \Lambda_{\lambda}^{x}$. It has been seen that $H_{\eta}^{x} \cap \Lambda_{\lambda}^{x}$ is always an horizontal closed interval, thus defining a mapping from the dual variable to the possible saddle strategies:

$$
(\eta, \lambda) \longmapsto\left[\alpha_{\min }^{\eta \lambda}, \alpha_{\max }^{\eta \lambda}\right] \times \varphi^{\eta \lambda} .
$$

But while this mapping is $1: 1$ for $\varphi$ (i.e. $\varphi^{\eta \lambda}$ ), it is characterized by a minimum value $\alpha_{\min }^{\eta \lambda}$ and a maximum value $\alpha_{\max }^{\eta \lambda}$ for $\alpha$. However, the case $\alpha_{\min }^{\eta \lambda}(x)=\alpha_{\max }^{\eta \lambda}(x)$ is quite common; in fact, because of the middle flatness of $\Lambda_{\lambda}^{x}$ and the two extremal flatness of $H_{\eta}^{x}$, there is at most two $\lambda$ such that $\alpha_{\min }^{\eta \lambda}(x)<\alpha_{\max }^{\eta \lambda}(x)$.

The crucial point is that $\alpha_{\min }^{\eta \lambda}, \alpha_{\max }^{\eta \lambda}$, and $\varphi^{\eta \lambda}$ are simply and entirely defined and computable from the problem data. However, the explicit definition of these functions is not given in the main part of this paper, because a lot of case checking is required. Readers should refer to Appendix A for more details.

Knowing $\alpha_{\min }^{\eta \lambda}, \alpha_{\max }^{\eta \lambda}$, and $\varphi^{\eta \lambda}$ it is useful to define the following global values:

$$
\begin{aligned}
\phi^{\eta \lambda}=\sum_{x \in E} \varphi^{\eta \lambda}(x), A_{\min }^{\eta \lambda}=\sum_{x \in E} \alpha_{\min }^{\eta \lambda}(x), & \\
& \text { and } A_{\max }^{\eta \lambda}=\sum_{x \in E} \alpha_{\max }^{\eta \lambda}(x) .
\end{aligned}
$$

The values $\phi^{\eta \lambda}, A_{\min }^{\eta \lambda}$, and $A_{\max }^{\eta \lambda}$ are of constant use in the development of our algorithm.
Variation of $\phi^{\eta \lambda}, A_{\min }^{\eta \lambda}$, and $A_{\max }^{\eta \lambda}$. Our interest now focuses on the variation of $\phi^{\eta \lambda}, A_{\min }^{\eta \lambda}$, and $A_{\max }^{\eta \lambda}$ according to the variables $\eta$ and $\lambda$. Most of the subsequent variation properties are foreseeable by considering the graphical properties of the curves $H_{\eta}^{x}$ and $\Lambda_{\lambda}^{x}$; this paragraph offers such qualitative graphical justification. A proof is possible however by means of the constraint inversion described in Appendix A (refer to Appendix B).

It appears that an increase of $\eta$ produces an upswelling (associated with a left shifting) of the curve $H_{\eta}^{x}$. Consequently, the increase of $\eta$ yields also an up-left move of the intersection $H_{\eta}^{x} \cap \Lambda_{\lambda}^{x}$, i.e.

$$
\eta_{1}<\eta_{2} \Longrightarrow\left\{\begin{array}{l}
\alpha_{\min }^{\eta_{1} \lambda}(x) \geq \alpha_{\min }^{\eta_{2} \lambda}(x) \\
\alpha_{\max }^{\eta_{1} \lambda}(x) \geq \alpha_{\max }^{\eta_{2} \lambda}(x) \\
\varphi^{\eta_{1} \lambda}(x) \leq \varphi^{\eta_{2} \lambda}(x)
\end{array}\right.
$$

This property is provable by means of the constraint inversion (see Appendices A and B).

An increase of $\lambda$ produces a left swelling (associated with a down shifting) of the curve $\Lambda_{\lambda}^{x}$, and implies consequently a down-left move of the intersection $H_{\eta}^{x} \cap \Lambda_{\lambda}^{x}$. Nevertheless, the intersection variation is somewhat sharper here:

$$
\lambda_{1}<\lambda_{2} \Longrightarrow\left\{\begin{array}{l}
\alpha_{\min }^{\eta \lambda_{1}}(x) \geq \alpha_{\max }^{\eta \lambda_{2}}(x) \\
\varphi^{\eta \lambda_{1}}(x) \geq \varphi^{\eta \lambda_{2}}(x)
\end{array}\right.
$$

This property is provable by means of the constraint inversion (Appendices A and B). The variation of the global variables are then deduced, yielding the following proposition:

PROPOSITION 2: Consider variations of dual variables $\eta$ and $\lambda$; then the variations of $A_{\min }^{\eta \lambda}, A_{\max }^{\eta \lambda}$, and $\Phi^{\eta \lambda}$ have 


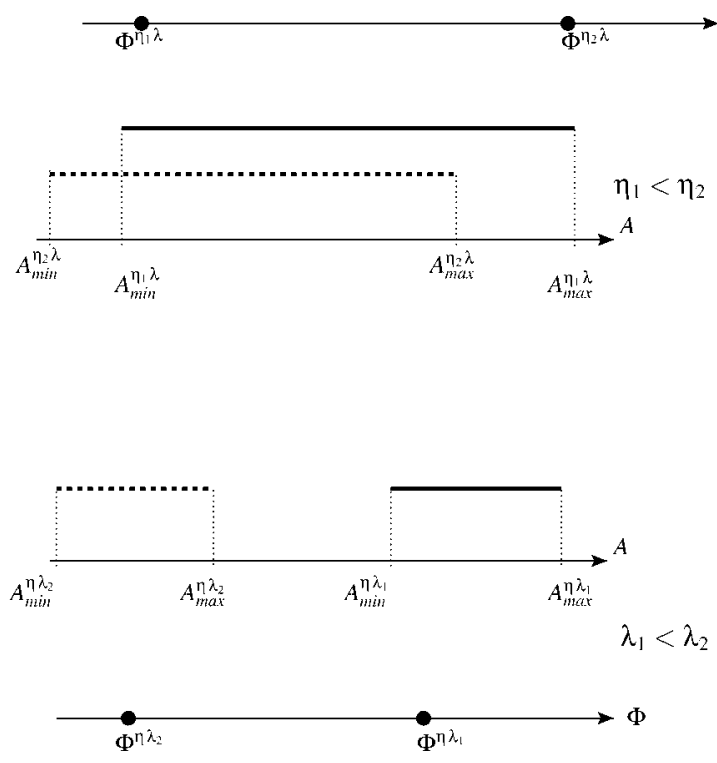

Figure 2. An illustration of Proposition 2.

monotonicity properties, as given by

$$
\forall \lambda, \quad \eta_{1}<\eta_{2} \Rightarrow\left\{\begin{array}{l}
A_{\min }^{\eta_{1} \lambda} \geq A_{\min }^{\eta_{2} \lambda} \\
A_{\max }^{\eta_{1} \lambda} \geq A_{\max }^{\eta_{2} \lambda} \\
\phi^{\eta_{1} \lambda} \leq \phi^{\eta_{2} \lambda}
\end{array}\right.
$$

and

$$
\forall \eta, \quad \lambda_{1}<\lambda_{2} \Rightarrow\left\{\begin{array}{l}
A_{\min }^{\eta \lambda_{1}} \geq A_{\max }^{\eta \lambda_{2}} \\
\phi^{\eta \lambda_{1}} \geq \phi^{\eta \lambda_{2}}
\end{array}\right.
$$

The above properties [see Eqs. (13) and (14) ] will be applied subsequently in order to compute the game value by means of a bisectional method. They are summarized in Fig. 2. In order to apply this method, some continuity properties are necessary.

Continuity. Let us begin by the following definitionproposition.

DEFINITION 1: Let $I \subset \mathbb{R}$ and $J \subset \mathbb{R}$. The distance $d(I, J)$ between $I$ and $J$ is classicaly defined by

$$
d(I, J)=\inf _{i \in I} \inf _{j \in J}|i-j| .
$$

Then, the following properties hold:

$$
\left\{\begin{array}{l}
(\eta, \lambda) \mapsto \varphi^{\eta \lambda}(x) \quad \text { is continuous, } \\
d\left(\left[\alpha_{\min }^{\eta \lambda}(x), \alpha_{\max }^{\eta \lambda}(x)\right],\left[\alpha_{\min }^{\eta^{\prime} \lambda^{\prime}}(x), \alpha_{\max }^{\eta^{\prime} \lambda^{\prime}}(x)\right]\right) \underset{\eta^{\prime}, \lambda^{\prime} \rightarrow \eta, \lambda}{\longrightarrow} 0, \\
\eta \mapsto\left(\alpha_{\min }^{\eta \lambda}(x), \alpha_{\max }^{\eta \lambda}(x)\right) \quad \text { is continuous for any } \lambda \geq 0 .
\end{array}\right.
$$

These properties are rather obvious, when considering the graphical properties of $H_{\eta}^{x}$ and $\Lambda_{\lambda}^{x}$. In particular,

- the curve $H_{\eta}^{x}$ is nondecreasing, but without discontinuity;

- since $p_{x}^{\prime}$ is continuous, $H_{\eta}^{x}$ evolves continuously with $\eta$;

- since $p_{x}$ is continuous, $\Lambda_{\lambda}^{x}$ evolves continuously with $\lambda$; and

- the curve $\Lambda_{\lambda}^{x}$ is nonincreasing.

The property Eq. (15) is provable by means of the constraint inversion (Appendices A and B ). Now, the continuity of the local variables implies a continuous behavior of the global variables:

$$
\left\{\begin{array}{l}
(\eta, \lambda) \mapsto \phi^{\eta \lambda} \quad \text { is continuous, } \\
d\left(\left[A_{\min }^{\eta \lambda}, A_{\max }^{\eta \lambda}\right],\left[A_{\min }^{\eta^{\prime} \lambda^{\prime}}, A_{\max }^{\left.\eta^{\eta^{\prime} \lambda^{\prime}}\right]}\right]\right) \underset{\eta^{\prime}, \lambda^{\prime} \rightarrow \eta, \lambda}{\longrightarrow} 0, \\
\eta \mapsto\left(A_{\min }^{\eta \lambda}, A_{\max }^{\eta \lambda}\right) \quad \text { is continuous for any } \lambda \geq 0 .
\end{array}\right.
$$

The continuity of the global variables will ensure that the bisectional processes work.

As a first step of the condition inversion, a bisectional process will be applied to the dual variable $\eta$ only, and $\eta$ will be expressed as a function of $\lambda$.

Implicit definition of $\eta(\lambda)$. The purpose of this paragraph is to characterize the function $\eta(\lambda)$, which is implicitly defined when the constraint $\sum_{x \in E} \varphi_{o}(x)=\phi_{o}$ is fulfilled. As a first step, a rough bounding of $\eta$ is derived. This bounding is used in order to initialize a bisectional method (described in the next section) for the computation of $\eta(\lambda)$.

Define:

$$
\eta_{\min }=\min _{x}\left(\alpha_{2}(x) p_{x}^{\prime}\left(\varphi_{1}(x)\right)\right)
$$

and

$$
\eta_{\max }=\max _{x}\left(\alpha_{1}(x) p_{x}^{\prime}\left(\varphi_{2}(x)\right)\right) .
$$

Definition (8) of $H_{\eta}$ implies

$$
\forall \lambda, \quad\left\{\begin{array}{l}
\eta \leq \eta_{\min } \Longrightarrow \varphi^{\eta \lambda}=\varphi_{1}, \\
\eta \geq \eta_{\max } \Longrightarrow \varphi^{\eta \lambda}=\varphi_{2} .
\end{array}\right.
$$

Thus, the function $\varphi^{\eta \lambda}$ is independent of the dual variables, when $\eta \leq \eta_{\min }$ or when $\eta \geq \eta_{\max }$. Owing to Definition (9) of $\Lambda_{\lambda}$, the bounds $\alpha_{\min }^{\eta \lambda}$ and $\alpha_{\max }^{\eta \lambda}$ then happen to be independent of the dual variable $\eta$, in the following meaning:

$\forall \lambda, \quad\left\{\begin{array}{c}{\left[\eta_{1} \leq \eta_{\min } \text { and } \eta_{2} \leq \eta_{\min }\right] \Longrightarrow\left(\varphi^{\eta_{1} \lambda}, \alpha_{\min }^{\eta_{1} \lambda}, \alpha_{\max }^{\eta_{1} \lambda}\right)} \\ =\left(\varphi^{\eta_{2} \lambda}, \alpha_{\min }^{\eta_{2} \lambda}, \alpha_{\max }^{\eta_{2} \lambda}\right), \\ {\left[\eta_{1} \geq \eta_{\max } \text { and } \eta_{2} \geq \eta_{\max }\right] \Longrightarrow\left(\varphi^{\eta_{1} \lambda}, \alpha_{\min }^{\eta_{1} \lambda}, \alpha_{\max }^{\eta_{1} \lambda}\right)} \\ =\left(\varphi^{\eta_{2} \lambda}, \alpha_{\min }^{\eta_{2} \lambda}, \alpha_{\max }^{\eta_{2} \lambda}\right) .\end{array}\right.$ 
In other words, every possible configuration for the game may be represented by a dual variable $\eta \in\left[\eta_{\min }, \eta_{\max }\right]$. For this reason, the function $\eta(\lambda)$ will be constructed within this range, subsequently. Then, related to the extremal duals $\eta_{\text {min }}$ and $\eta_{\max }$ are also defined the extremal global values of the resources:

$$
\phi^{\eta_{\min }}=\phi^{\eta_{\min } \lambda}=\sum_{x \in E} \varphi_{1}(x) \text { and } \phi^{\eta_{\max }}=\phi^{\eta_{\max } \lambda}=\sum_{x \in E} \varphi_{2}(x) .
$$

From Eq. (13), it appears that $\eta \mapsto \phi^{\eta \lambda}$ is non decreasing. The bounds $\eta_{\min }$ and $\eta_{\max }$ being defined, it seems possible to derive $\eta(\lambda)$ by inverting the equation $\phi^{\eta \lambda}=\phi_{o}$ and using a bisectional process. This process is guaranteed to work, since $\eta \mapsto \phi^{\eta \lambda}$ is continuous [Eq. (16)].

It follows that every $\phi \in\left[\sum_{x \in E} \varphi_{1}(x), \sum_{x \in E} \varphi_{2}(x)\right]$ admits a nonempty connected set of antecedents. It is in particular true for $\phi_{o}$. The set of antecedents is often reduced to one element, when the mapping $\eta \mapsto \phi^{\eta \lambda}$ is increasing, otherwise it is an interval when the mapping $\eta \mapsto \phi^{\eta \lambda}$ is flat. Thus, the bounds $\eta_{\min }(\lambda) \in\left[\eta_{\min }, \eta_{\max }\right]$ and $\eta_{\max }(\lambda) \in\left[\eta_{\min }, \eta_{\max }\right]$ are defined such that

$$
\forall \eta \in\left[\eta_{\min }, \eta_{\max }\right], \quad \phi^{\eta \lambda}=\phi_{o} \Leftrightarrow \eta \in\left[\eta_{\min }(\lambda), \eta_{\max }(\lambda)\right],
$$

and the function $\eta(\lambda)$ is thus defined by $\eta(\lambda)=\left[\eta_{\min }, \eta_{\max }\right]$. These bounds will be actually computed by the bisectional process over $\eta$. However, in order to complete the conditions for inversion, a second bisectional process (described in the next section) will be applied to the variable $\lambda$. This second process will tune $\lambda$ toward the constraint $\sum_{x \in E} \alpha_{o}(x)=A_{o}$. In order to justify this bisectional process, it is necessary to verify that $\lambda \mapsto A^{\eta(\lambda) \lambda}$ is "monotonic." This is the last purpose of the paragraph.

Assume $\lambda_{1}<\lambda_{2}$. Then, it is deduced $\phi^{\eta_{\min }\left(\lambda_{2}\right) \lambda_{1}} \geq$ $\phi^{\eta_{\min }\left(\lambda_{2}\right) \lambda_{2}}$ from Eq. (14). Since by definition $\phi^{\eta_{\min }\left(\lambda_{2}\right) \lambda_{2}}=$ $\phi_{o}=\phi^{\eta_{\min }\left(\lambda_{1}\right) \lambda_{1}}$, the property $\phi^{\eta_{\min }\left(\lambda_{2}\right) \lambda_{1}} \geq \phi^{\eta_{\min }\left(\lambda_{1}\right) \lambda_{1}}$ holds. Now, $\eta \mapsto \phi^{\eta \lambda_{1}}$ is a non decreasing function [see eq. (13)] and it is deduced:

$$
\eta_{\min }\left(\lambda_{2}\right)>\eta_{\min }\left(\lambda_{1}\right) \quad \text { or } \quad \phi^{\eta_{\min }\left(\lambda_{2}\right) \lambda_{1}}=\phi^{\eta_{\min }\left(\lambda_{1}\right) \lambda_{1}}
$$

Since $\eta_{\min }\left(\lambda_{1}\right)$ is defined as the smaller $\eta$ such that $\phi^{\eta \lambda_{1}}=\phi_{o}$, we have

$$
\eta_{\min }\left(\lambda_{2}\right)>\eta_{\min }\left(\lambda_{1}\right) \quad \text { or } \quad \eta_{\min }\left(\lambda_{2}\right) \geq \eta_{\min }\left(\lambda_{1}\right)
$$

Finally, it has been shown that $\eta_{\min }\left(\lambda_{2}\right) \geq \eta_{\min }\left(\lambda_{1}\right)$ and it is similarly proved that $\eta_{\max }\left(\lambda_{2}\right) \geq \eta_{\max }\left(\lambda_{1}\right)$. The variations of $\eta_{\min }(\lambda)$ and $\eta_{\max }(\lambda)$ are thus monotonic:

$$
\lambda_{1}<\lambda_{2} \Longrightarrow\left\{\begin{array}{l}
\eta_{\min }\left(\lambda_{1}\right) \leq \eta_{\min }\left(\lambda_{2}\right) \\
\eta_{\max }\left(\lambda_{1}\right) \leq \eta_{\max }\left(\lambda_{2}\right)
\end{array}\right.
$$

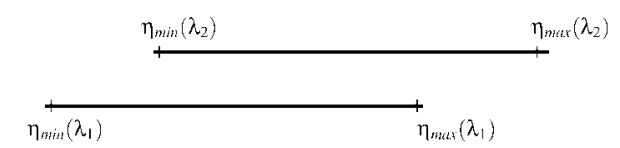

$\lambda_{1}<\lambda_{2}$
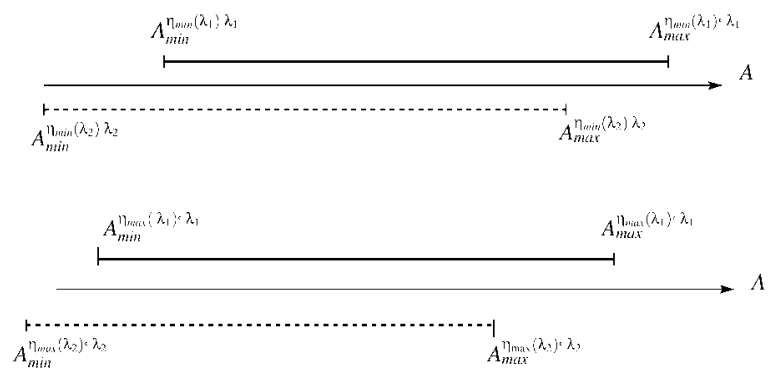

Figure 3. An illustration of Proposition 3.

Now, $A_{\min }^{\eta \lambda}$ and $A_{\max }^{\eta \lambda}$ are decreasing for both $\eta$ and $\lambda$ (Properties (13) and (14)) and the following properties are derived:

PROPOSITION 3: Consider two values $\left(\lambda_{1}\right.$ and $\left.\lambda_{2}\right)$ of the parameter $\lambda$, with $\lambda_{1}<\lambda_{2}$; then the following inequalities hold true:

$$
\lambda_{1}<\lambda_{2} \Longrightarrow\left\{\begin{array}{l}
A_{\min }^{\eta_{\min }\left(\lambda_{1}\right) \lambda_{1}} \geq A_{\min }^{\eta_{\min }\left(\lambda_{2}\right) \lambda_{2}} \\
A_{\max }^{\eta_{\min }\left(\lambda_{1}\right) \lambda_{1}} \geq A_{\max }^{\eta_{\min }\left(\lambda_{2}\right) \lambda_{2}} \\
A_{\min }^{\eta_{\max }\left(\lambda_{1}\right) \lambda_{1}} \geq A_{\operatorname{mix}}^{\eta_{\max }\left(\lambda_{2}\right) \lambda_{2}} \\
A_{\max }^{\eta_{\max }\left(\lambda_{1}\right) \lambda_{1}} \geq A_{\max }^{\eta_{\max }\left(\lambda_{2}\right) \lambda_{2}}
\end{array}\right.
$$

These properties are graphically illustrated by Fig. 3: It is now possible to describe the entire inversion algorithm.

\section{ALGORITHM}

Because of the monotonic variation of $A_{\min }^{\eta \lambda}, A_{\max }^{\eta \lambda}$, and $\phi^{\eta \lambda}$, and the continuity property (16), bisectional methods are chosen for the inversion conditions. Our algorithm is made of three parts. The first part computes the optimal dual parameter $\lambda_{o}$. The second part sharpens the convergence and improves some subconditions, by adjusting the optimal dual parameter $\eta_{o}$. At this point, the convergence is almost achieved. The last part makes a final tuning of $\alpha$, so as to equalize to $A_{o}$ and reduce some indetermination.

Computing $\lambda_{o}$. First it is necessary to define the subprocesses, which will compute $\eta_{\min }(\lambda)$ and $\eta_{\max }(\lambda)$ : thanks to the non decreasing property of $\eta \mapsto \phi^{\eta \lambda}$, two bisectional processes around $\phi_{o}$ are used in order to compute $\eta_{\min }(\lambda)$ and $\eta_{\max }(\lambda)$.

The main part of the process consists in finding $\lambda$ such that $A_{o} \in\left[A_{\min }^{\eta_{\max }(\lambda) \lambda}, A_{\max }^{\eta_{\min }(\lambda) \lambda}\right]$. Thanks to the non increasing 


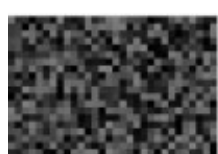

$\alpha_{1}$

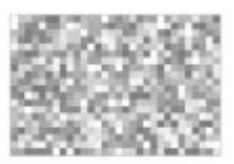

$\alpha_{2}$

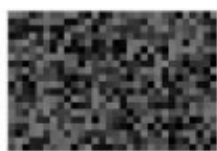

$\varphi_{1}$

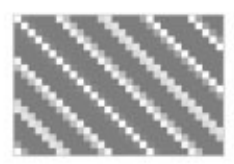

$\varphi_{2}$

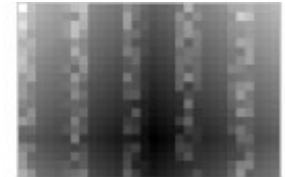

Visibility parameter $\omega$

Figure 4. Game description.

property (see Proposition 3), this task is done by a bisectional process. However, this process will call the subprocesses computing $\eta_{\min }(\lambda)$ and $\eta_{\max }(\lambda)$, and thus constitutes a double bisectional method.

After this stage, the optimal dual variable $\lambda_{o}$ is computed.

Computing $\eta_{o}$. It is noteworthy that $\phi^{\eta \lambda_{o}}=\phi_{o}$ for any $\eta \in\left[\eta_{\min }\left(\lambda_{o}\right), \eta_{\max }\left(\lambda_{o}\right)\right]$; we do not have to care about the constraint on $\phi_{o}$ anymore. As a consequence, the computation of $\eta_{o}$ will be obtained by applying the constraint related to $A_{o}$.

Now, since $A_{o} \in\left[A_{\min }^{\eta_{\max }\left(\lambda_{o}\right) \lambda_{o}}, A_{\max }^{\eta_{\min }\left(\lambda_{o}\right) \lambda_{o}}\right]$, there is $\eta \in\left[\eta_{\min }\left(\lambda_{o}\right)\right.$, $\left.\eta_{\max }\left(\lambda_{o}\right)\right]$ such that $A_{o} \in\left[A_{\min }^{\eta \lambda_{o}}, A_{\max }^{\eta \lambda_{o}}\right]$. This $\eta$ will be the optimal dual variable $\eta_{o}$. To compute it, a bisectional process is again instrumental, owing to the nonincrease property (13) of $\eta \mapsto A_{\min }^{\eta \lambda_{o}}$ and $\eta \mapsto A_{\max }^{\eta \lambda_{o}}$.

Refinement. The optimal dual variables $\eta_{o}$ and $\lambda_{o}$ are now computed and the function $\varphi_{o}$ is defined as $\varphi^{\eta_{o} \lambda_{o}}$. However, some indetermination is possible for $\alpha_{o}$, when $A_{\text {min }}^{\eta_{o} \lambda_{o}}<$ $A_{\mathrm{max}}^{\eta_{o} \lambda_{o}}$. Whatever, the objective is to fulfill the last constraint $\sum_{x \in E} \alpha_{o}(x)=A^{o}$. Recalling that $A_{\min }^{\eta_{o} \lambda_{o}}=\sum_{x \in E} \alpha_{\min }^{\eta_{o} \lambda_{o}}(x)$ and $A_{\max }^{\eta_{o} \lambda_{o}}=\sum_{x \in E} \alpha_{\max }^{\eta_{o} \lambda_{o}}(x)$, an optimal target strategy $\alpha_{o}$ could be defined as the barycenter of $\alpha_{\min }^{\eta_{o} \lambda_{o}}$ and $\alpha_{\max }^{\eta_{o} \lambda_{o}}$, where the weights are given by the relative positions of $A_{\min }^{\eta_{o} \lambda_{o}}, A_{\max }^{\eta_{o} \lambda_{o}}$, and $A_{o}$ :

$$
\left\{\begin{array}{l}
\varphi_{o}=\varphi^{\eta_{o} \lambda_{o}}, \\
\alpha_{o}=\alpha_{\min }^{\eta_{o} \lambda_{o}}+\frac{A_{o}-A_{\min }^{\eta_{o} \lambda_{o}}}{A_{\max }^{\eta_{o} \lambda_{o}}-A_{\min }^{\eta_{o} \lambda_{o}}}\left(\alpha_{\max }^{\eta_{o} \lambda_{o}}-\alpha_{\min }^{\eta_{o} \lambda_{o}}\right) .
\end{array}\right.
$$

Notice that there may be many other choices for $\alpha_{o}$.

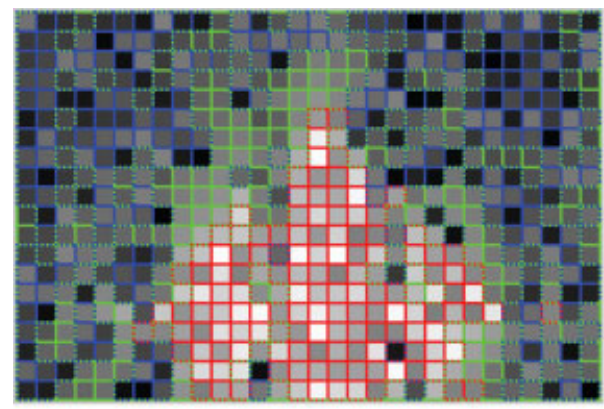

$\alpha_{O}$
The whole process. The whole process is summed up below:

i. Find $\lambda_{o}$ such that $A_{o} \in\left[A_{\min }^{\eta_{\max }\left(\lambda_{o}\right) \lambda_{o}}, A_{\max }^{\eta_{\min }\left(\lambda_{o}\right) \lambda_{o}}\right]$; do it by means of a bisectional process; a sub-procedure is used to compute $\eta_{\min }(\lambda)$ and $\eta_{\max }(\lambda)$.

ii. Find $\eta_{o}$, element of $\left[\eta_{\min }\left(\lambda_{o}\right), \eta_{\max }\left(\lambda_{o}\right)\right]$, such that $A_{o} \in\left[A_{\min }^{\eta_{o} \lambda_{o}}, A_{\max }^{\eta_{o} \lambda_{o}}\right]$; do it by means of a bisectional process.

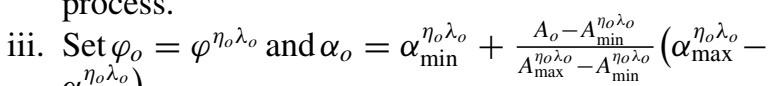
$\left.\alpha_{\min }^{\eta_{o} \lambda_{o}}\right)$

Subprocesses. Compute $\eta_{\min }(\lambda)$ and $\eta_{\max }(\lambda)$ by means of a bisectional process.

\section{RESULTS}

In the sequel, the search space $E$ is a set of $30 \times 20$ cells. The values $A_{o}=1$ and $\phi_{o}=30$ are used.

\subsection{Some Examples}

In this section, a game example is solved by our algorithm. It is characterized by the local bounds $\alpha_{1}, \alpha_{2}, \varphi_{1}$, and $\varphi_{2}$ described in Fig. 4; in this figure and the following, the dark cells are representing low values, while the bright cells represent high values. The conditional probability, $p$, is of exponential form $p_{x}(\varphi)=\exp \left(-\omega_{x} \varphi\right)$. The visibility parameter $\omega_{x}$ is weak for poor detection and high for good detection. The parameter $\omega$ is described in Fig. 4. Optimal functions $\alpha_{o}$ and $\varphi_{o}$ obtained via the algorithm are represented in Fig. 5. Again, the dark cells represent low values whereas

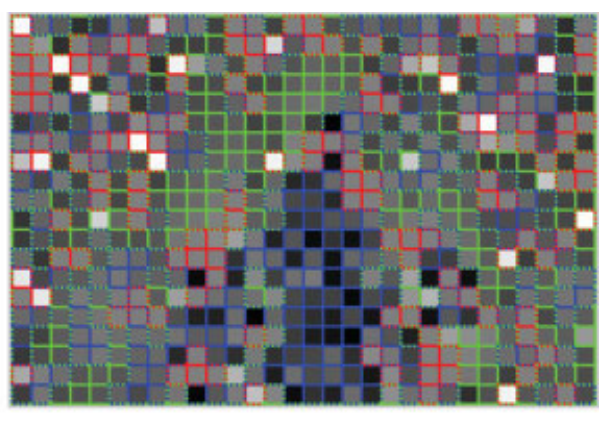

$\varphi_{O}$

Figure 5. Strategies. [Color figure can be viewed in the online issue, which is available at www.interscience.wiley.com.] 


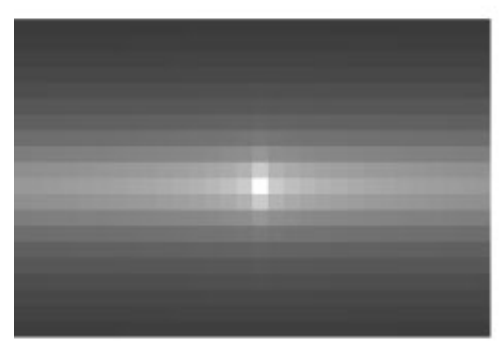

Prior $\bar{\alpha}$

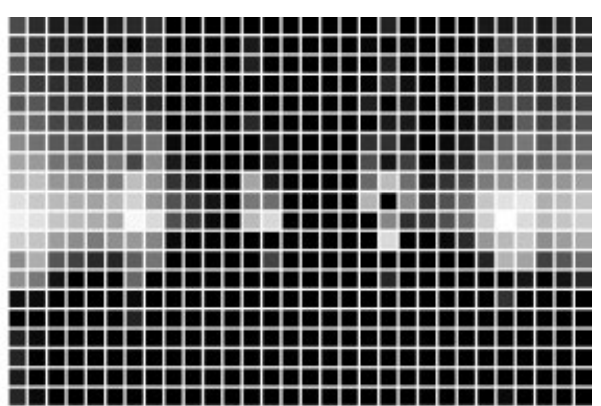

$\varphi_{o}$

Figure 6. Optimization of de Guenin ( $\alpha=\bar{\alpha}$ is fixed).

the bright cells represent high values. Moreover, the color of the cell boundaries indicates whether the bounds are reached or not. More precisely, a blue boundary on cell $x$ means $\varphi_{o}(x)=\varphi_{1}(x)$ or $\alpha_{o}(x)=\alpha_{1}(x)$. A green boundary on cell $x$ means $\varphi_{1}(x)<\varphi_{o}(x)<\varphi_{2}(x)$ or $\alpha_{1}(x)<\alpha_{o}(x)<\alpha_{2}(x)$. And a red boundary on cell $x$ means $\varphi_{o}(x)=\varphi_{2}(x)$ or $\alpha_{o}(x)=\alpha_{2}(x)$. Fig. 5 appears as a mix of the local bounding constraints and of the visibility parameters. It is noteworthy that $\alpha_{o}$ reaches its upper bound on cells with weak visibility (center of the search space) and its lower bound on cells with strong visibility (corners). The situation is opposite for $\varphi_{o}$.

Implementation. In this case, the convergence occurs after 483 iterations of the inner dichotomy (computation of $\eta(\lambda)$ ). The algorithm has been tested with its graphical user interface. The convergence time is about 2 on a SUN computer, using a single CPU "UltraSPARC-II" that runs at $360 \mathrm{MHz}$; the convergence criterion was strong (a precision of $10^{-6}$ ).

\subsection{A Comparison with de Guenin and Nakai}

Since the constrained games considered in this paper are middle links between a simple optimization (de Guenin) and a pure game (Nakai), it is interesting to make a comparison between these three viewpoints by means of correlated optimization/game examples.

Setting. In order to make the examples easily readable, the bounding constraints are removed for $\varphi$. However, the bounds are maintained for $\alpha$. More precisely, the bounds are absolute constraints for an optimization of de Guenin, i.e. $\alpha_{1}=\alpha_{2}$. Then, the bounds are gradually relaxed, i.e. $\alpha_{2}-\alpha_{1}$ increases, until vanishing. Vanished bounds, i.e. $\alpha_{1}=0$ and $\alpha_{2} \geq A_{o}$, make the constrained game equivalent to a pure game. Six such games, gradually relaxed, have been considered. All games are based on the same visibility $\omega_{x}$, as described in Fig. 4.

The first "game" is such that $\alpha_{1}=\alpha_{2}=\bar{\alpha}$, where in the prior $\bar{\alpha}$ is described by Fig. 6 . This case is equivalent to an optimization of de Guenin, and the optimized value $\varphi_{o}$ is described by Fig. 6 .

The subsequent games, with respective relaxation degrees $d \in\{0,1,2,3, \infty\}$, are defined with the bounds $\alpha_{1}^{d}=2^{-d} \bar{\alpha}_{1}$ and $\alpha_{2}^{d}=2^{d} \bar{\alpha}_{2}$, where $\bar{\alpha}_{1}$ and $\bar{\alpha}_{2}$ are two moderate distortions of the law $\bar{\alpha}$, such that $\bar{\alpha}_{1}<\bar{\alpha}<\bar{\alpha}_{2}$. The functions $\bar{\alpha}_{1}$ and $\bar{\alpha}_{2}$ are represented in Fig. 7. For each game, the computed saddle strategies are represented in Fig. 8.

Comments. For the small relaxation degree 0, it appears that the optimal target strategy $\alpha_{o}$ is still similar to $\bar{\alpha}$. The same result holds for the strategy $\varphi_{o}$, which appears to be quite similar to the solution of the simple optimization. But with the increase in the relaxation, the target optimal strategy focuses toward the lower center of the search space. As a consequence, the observer follows by putting more resources toward the center, although the visibility is bad. At last, the fully relaxed game (i.e. Nakai's game) is solved by an almost

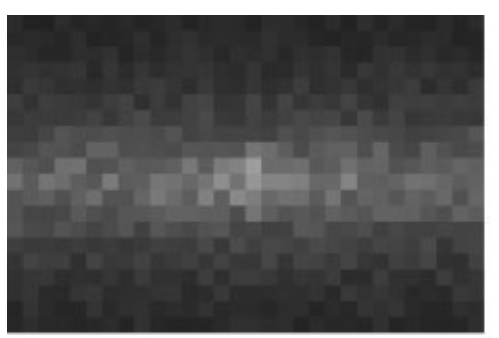

$\bar{\alpha}_{1}$

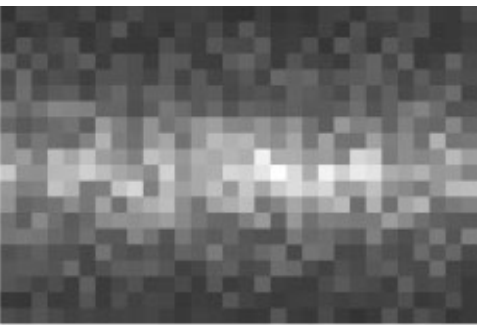

$\bar{\alpha}_{2}$

Figure 7. Bound prototypes for $\alpha$. 


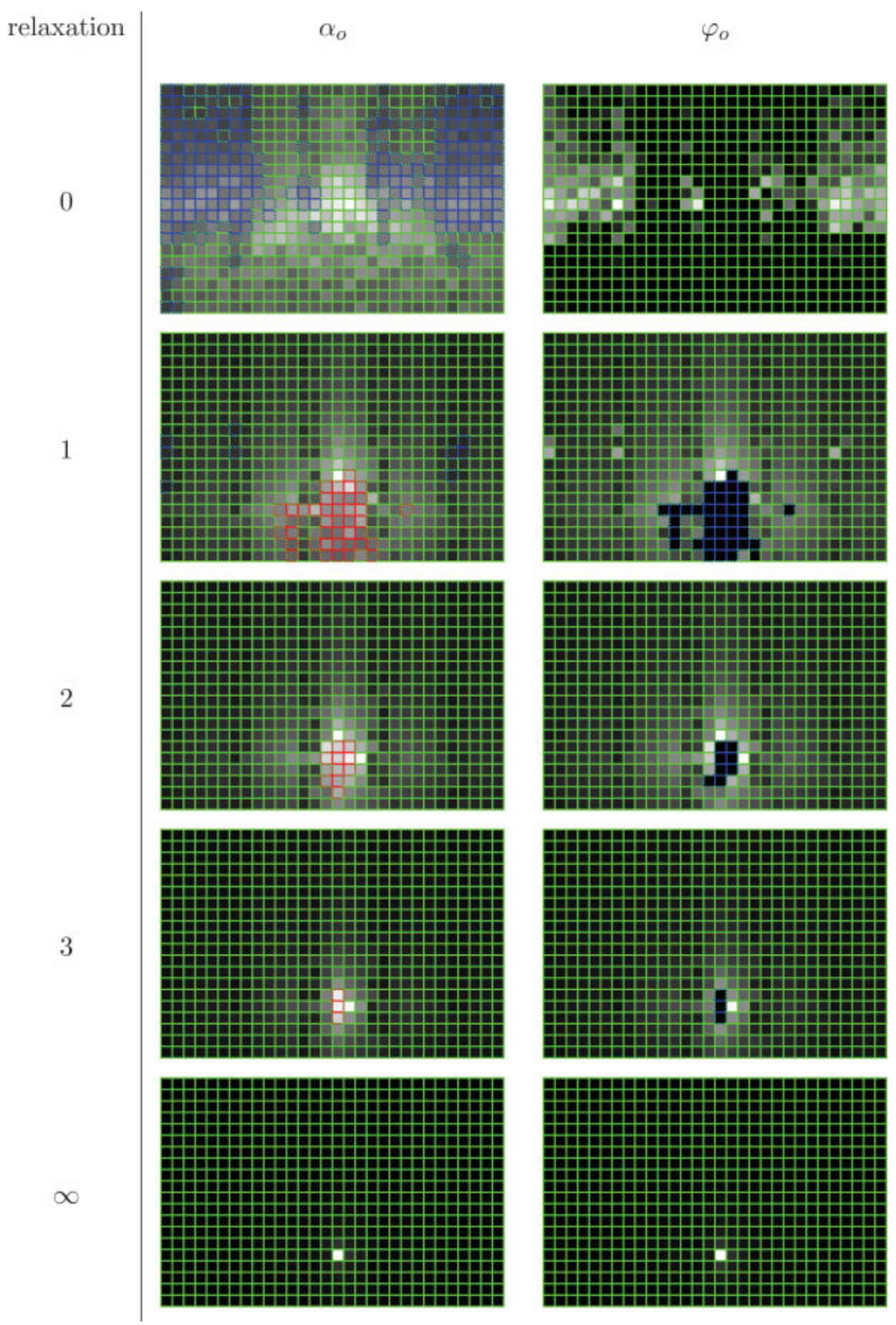

Figure 8. Strategies with constraints relaxation on $\alpha$. [Color figure can be viewed in the online issue, which is available at www.interscience.wiley.com.]

pure saddle point (the strategy $\alpha_{o}$ and thus $\varphi_{o}$ almost reduce to a point.). Notice however that Nakai's games are not solved by pure saddle points in general.

Some additional comments could be made about the colors associated with the strategies in Fig. 8. The main color is green: for most cells, the bounds are not reached, resulting in a "true" saddle point. However, the blue $(d=0$, left and right borders) and the red ( $d=1,2,3$, lower center) associated with $\alpha^{o}$ have a particular meaning. In the case of degree 0 , the lower bound of $\alpha$ is very high and implies 


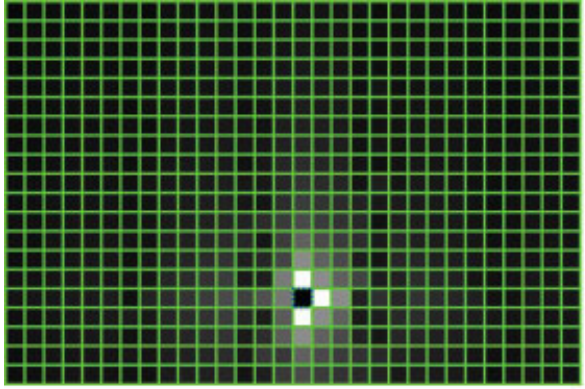

$\alpha_{o}$

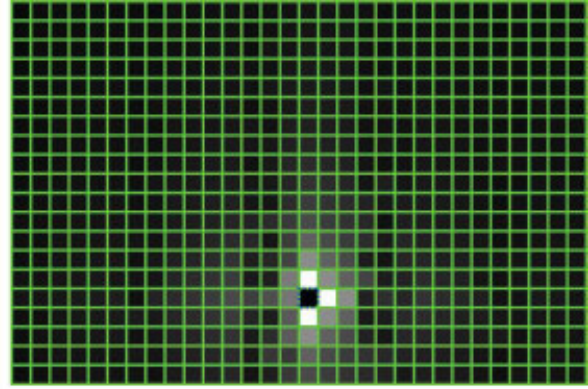

$\varphi_{o}$

Figure 9. No constraint on $\alpha+$ worse case exclusion. [Color figure can be viewed in the online issue, which is available at www.interscience.wiley.com.]

the most significant constraint against the target: the target is forced to play in the most visible areas. The target will minimize such play (blue color). In the cases $d=1,2,3$, the lower bound of $\alpha$ is rather small, but the upper bound is too limitative in the less visible areas: the target is not allowed to play here as much as it wants (red color). Conversely, the lower bound is reached by $\varphi^{o}$ on the same areas for $d=1,2,3$ (lower center).

Pure game. In the previous example, the optimal strategies of the pure game focused on a single cell, $(15,15)$. The reason is that the visibility for $(15,15)$ was $10 \times$ lower than for the other cells. In Fig. 9, the same game is optimized, but the cell $(15,15)$ is removed from the play, i.e. $\mathbb{T}=E \backslash\{(15,15)\}$. It appears that the strategies are no more focusing on single cells.

\section{CONCLUSION}

Our aim was to solve a spatial resource allocation problem, in a game context between the target and the searcher. A great enhancement and a generalization of both Nakai's game and de Guenin's optimization were obtained, by means of games with constraints on the mixed strategies. This viewpoint is versatile, allowing subtle modelling of the target and resource behavior. It is not limited to simple priors on the available target location. The algorithm developed in this work is efficient and is theoretically grounded.

A more general study of such games with constraints on the mixed strategies should be investigated in the future. These games are quite interesting, because they are related to a particular modelling of the prior knowledge in an optimization problem. More precisely, an objective criterion for optimizing a function, when the prior knowledge is probabilist (e.g. de Guenin's problem), is the optimization of the function mean. This is a purely uncertainty-based prior. When the prior is defined by a set of possible configurations of the variable, the objective criterion is generally a (classical) game (e.g. Nakai's problem). This is a purely imprecision-based prior.
There are intermediate models for defining the prior about the knowledge. For example, it is possible to define probabilistic laws on imprecision intervals. Such models are a mix of both uncertainty and imprecision. Our paper investigated a particular case of mixed model. The definition of an objective optimization criterion related to a mixed prior modelling is still an uneasy task. Games with constraints on the mixed strategies could be good candidates.

\section{APPENDIX A: COMPUTING $\alpha_{\min }^{\eta \lambda}, \alpha_{\max }^{\eta \lambda}, \operatorname{AND} \varphi^{\eta \lambda}$}

The purpose of this section is to give a systematic method for computing $\alpha_{\min }^{\eta \lambda}, \alpha_{\max }^{\eta \lambda}$ and $\varphi^{\eta \lambda}$, when duals $\eta$, and $\lambda$ are given (proofs are left to the reader). Theoretically, this problem is very simple, but the implementation is not easy because a lot of cases have to be checked.

Let $\eta<0, \lambda \geq 0$, and $x \in E$. Five main cases are considered.

CASE I: $p_{x}^{-1}(\lambda)<\varphi_{1}(x)$.

$\alpha_{\min }^{\eta \lambda}(x)$ and $\alpha_{\max }^{\eta \lambda}(x)$ are directly stated:

$$
\alpha_{\min }^{\eta \lambda}(x)=\alpha_{\max }^{\eta \lambda}(x)=\alpha_{1}(x)
$$

For $\varphi^{\eta \lambda}(x)$, three subcases are considered:

$$
\begin{aligned}
& \text { CASE a: } \alpha_{1}(x)<\frac{\eta}{p_{x}^{\prime}\left(\varphi_{1}(x)\right)} \text {. Then } \varphi^{\eta \lambda}(x)=\varphi_{1}(x) . \\
& \text { CASE b: } \frac{\eta}{p_{x}^{\prime}\left(\varphi_{1}(x)\right)} \leq \alpha_{1}(x) \leq \frac{\eta}{p_{x}^{\prime}\left(\varphi_{2}(x)\right)} \text {. Then } \varphi^{\eta \lambda}(x)=p_{x}^{\prime-1}\left(\frac{\eta}{\alpha_{1}(x)}\right) . \\
& \text { CASE c: } \alpha_{1}(x)>\frac{\eta}{p_{x}^{\prime}\left(\varphi_{2}(x)\right)} \text {. Then } \varphi^{\eta \lambda}(x)=\varphi_{2}(x) .
\end{aligned}
$$

CASE II: $p_{x}^{-1}(\lambda)=\varphi_{1}(x)$.

Four subcases are considered here:

$$
\begin{aligned}
\text { CASE a: } & \frac{\eta}{p_{x}^{\prime}\left(\varphi_{1}(x)\right)}>\alpha_{2}(x) . \text { Then } \varphi^{\eta \lambda}(x)=\varphi_{1}(x), \alpha_{\min }^{\eta \lambda}(x)=\alpha_{1}(x), \\
\text { and } \alpha_{\max }^{\eta \lambda}(x)=\alpha_{2}(x) . & \\
\text { CASE b: } & \alpha_{1}(x) \leq \frac{\eta}{p_{x}^{\prime}\left(\varphi_{1}(x)\right)} \leq \alpha_{2}(x) . \text { Then } \varphi^{\eta \lambda}(x)=\varphi_{1}(x), \alpha_{\min }^{\eta \lambda}(x)= \\
& \alpha_{1}(x), \text { and } \alpha_{\max }^{\eta \lambda}(x)=\frac{\eta}{p_{x}^{\prime}\left(\varphi_{1}(x)\right)} . \\
\text { CASE c: } & \frac{\eta}{p_{x}^{\prime}\left(\varphi_{1}(x)\right)}<\alpha_{1}(x) \leq \frac{\eta}{p_{x}^{\prime}\left(\varphi_{2}(x)\right)} . \text { Then } \alpha_{\min }^{\eta \lambda}(x)=\alpha_{\max }^{\eta \lambda}(x)= \\
& \alpha_{1}(x) \text { and } \varphi^{\eta \lambda}(x)=p_{x}^{\prime-1}\left(\frac{\eta}{\alpha_{1}(x)}\right) . \\
\text { CASE d: } & \frac{\eta}{p_{x}^{\prime}\left(\varphi_{2}(x)\right)}<\alpha_{1}(x) . \text { Then } \varphi^{\eta \lambda}(x)=\varphi_{2}(x) \text { and } \alpha_{\min }^{\eta \lambda}(x)= \\
& \alpha_{\max }^{\eta \lambda}(x)=\alpha_{1}(x) .
\end{aligned}
$$

Naval Research Logistics DOI 10.1002/nav 
CASE III: $\varphi_{1}(x)<p_{x}^{-1}(\lambda)<\varphi_{2}(x)$.

First, define $\varphi_{L}$ and $\varphi_{R}$ by

$$
\left\{\begin{array}{l}
\alpha_{1}(x)<\frac{\eta}{p_{x}^{\prime}\left(\varphi_{1}(x)\right)} \Rightarrow \varphi_{L}=\varphi_{1}(x), \\
\frac{\eta}{p_{x}^{\prime}\left(\varphi_{1}(x)\right)} \leq \alpha_{1}(x) \leq \frac{\eta}{p_{x}^{\prime}\left(\varphi_{2}(x)\right)} \Rightarrow \varphi_{L}=p_{x}^{\prime-1}\left(\frac{\eta}{\alpha_{1}(x)}\right), \\
\alpha_{1}(x)>\frac{\eta}{p_{x}^{\prime}\left(\varphi_{2}(x)\right)} \Rightarrow \varphi_{L}=\varphi_{2}(x),
\end{array}\right.
$$

and

$$
\left\{\begin{array}{l}
\alpha_{2}(x)>\frac{\eta}{p_{x}^{\prime}\left(\varphi_{2}(x)\right)} \Rightarrow \varphi_{R}=\varphi_{2}(x), \\
\frac{\eta}{p_{x}^{\prime}\left(\varphi_{1}(x)\right)} \leq \alpha_{2}(x) \leq \frac{\eta}{p_{x}^{\prime}\left(\varphi_{2}(x)\right)} \Rightarrow \varphi_{R}=p_{x}^{\prime}-1\left(\frac{\eta}{\alpha_{2}(x)}\right), \\
\alpha_{2}(x)<\frac{\eta}{p_{x}^{\prime}\left(\varphi_{1}(x)\right)} \Rightarrow \varphi_{R}=\varphi_{1}(x) .
\end{array}\right.
$$

Then, three cases are considered:

CASE a: $\varphi_{L}>p_{x}^{-1}(\lambda) . \quad$ Then $\quad \alpha_{\min }^{\eta \lambda}(x)=\alpha_{\max }^{\eta \lambda}(x)=\alpha_{1}(x) \quad$ and $\varphi^{\eta \lambda}(x)=\varphi_{L}$

CASE b: $\varphi_{L} \leq p_{x}^{-1}(\lambda) \leq \varphi_{R}$. Then $\alpha_{\min }^{\eta \lambda}(x)=\alpha_{\max }^{\eta \lambda}(x)=\frac{\eta}{p_{x}^{\prime}\left(p_{x}^{-1}(\lambda)\right)}$ and $\varphi^{\eta \lambda}(x)=p_{x}^{-1}(\lambda)$.

CASE c: $\varphi_{R}<p_{x}^{-1}(\lambda)$. Then $\quad \alpha_{\min }^{\eta \lambda}(x)=\alpha_{\max }^{\eta \lambda}(x)=\alpha_{2}(x) \quad$ and $\varphi^{\eta \lambda}(x)=\varphi_{R}$

CASE IV: $p_{x}^{-1}(\lambda)=\varphi_{2}(x)$.

Four subcases are considered here:

$$
\begin{aligned}
\text { CASE a: } & \frac{\eta}{p_{x}^{\prime}\left(\varphi_{1}(x)\right)}>\alpha_{2}(x) . \text { Then } \varphi^{\eta \lambda}(x)=\varphi_{1}(x) \text { and } \alpha_{\min }^{\eta \lambda}(x)= \\
& \alpha_{\max }^{\eta \lambda}(x)=\alpha_{2}(x) . \\
\text { CASE b: } & \frac{\eta}{p_{x}^{\prime}\left(\varphi_{1}(x)\right)} \leq \alpha_{2}(x)<\frac{\eta}{p_{x}^{\prime}\left(\varphi_{2}(x)\right)} \text {. Then } \alpha_{\min }^{\eta \lambda}(x)=\alpha_{\max }^{\eta \lambda}(x)= \\
& \alpha_{2}(x) \text { and } \varphi^{\eta \lambda}(x)=p_{x}^{\prime-1}\left(\frac{\eta}{\alpha_{2}(x)}\right) . \\
\text { CASE c: } & \alpha_{1}(x) \leq \frac{\eta}{p_{x}^{\prime}\left(\varphi_{2}(x)\right)} \leq \alpha_{2}(x) . \text { Then } \varphi^{\eta \lambda}(x)=\varphi_{2}(x), \alpha_{\min }^{\eta \lambda}(x)= \\
& \frac{\eta}{p_{x}^{\prime}\left(\varphi_{2}(x)\right)}, \text { and } \alpha_{\max }^{\eta \lambda}(x)=\alpha_{2}(x) . \\
\text { CASE d: } & \frac{\eta}{p_{x}^{\prime}\left(\varphi_{2}(x)\right)}<\alpha_{1}(x) . \text { Then } \varphi^{\eta \lambda}(x)=\varphi_{2}(x), \alpha_{\min }^{\eta \lambda}(x)=\alpha_{1}(x), \\
& \text { and } \alpha_{\max }^{\eta \lambda}(x)=\alpha_{2}(x) .
\end{aligned}
$$

CASE V: $p_{x}^{-1}(\lambda)>\varphi_{2}(x)$.

$\alpha_{\min }^{\eta \lambda}(x)$ and $\alpha_{\max }^{\eta \lambda}(x)$ are directly stated:

$$
\alpha_{\min }^{\eta \lambda}(x)=\alpha_{\max }^{\eta \lambda}(x)=\alpha_{2}(x)
$$

For $\varphi^{\eta \lambda}(x)$, three subcases are considered:

$$
\begin{aligned}
& \text { CASE a: } \alpha_{2}(x)<\frac{\eta}{p_{x}^{\prime}\left(\varphi_{1}(x)\right)} \text {. Then } \varphi^{\eta \lambda}(x)=\varphi_{1}(x) . \\
& \text { CASE b: } \frac{\eta}{p_{x}^{\prime}\left(\varphi_{1}(x)\right)} \leq \alpha_{2}(x) \leq \frac{\eta}{p_{x}^{\prime}\left(\varphi_{2}(x)\right)} \text {. Then } \varphi^{\eta \lambda}(x)=p_{x}^{\prime-1}\left(\frac{\eta}{\alpha_{2}(x)}\right) . \\
& \text { CASE c: } \alpha_{2}(x)>\frac{\eta}{p_{x}^{\prime}\left(\varphi_{2}(x)\right)} \text {. Then } \varphi^{\eta \lambda}(x)=\varphi_{2}(x) .
\end{aligned}
$$

\section{APPENDIX B: PROOFS}

In this section, it is shown that the inversion of Appendix A implies the Propositions (11), (12), and (15). Since there are many cases, most verifications are left to the reader.

Naval Research Logistics DOI 10.1002/nav
Variation according to $\eta$. First, let us consider in Appendix A the Cases I, II, IV, and V. In all these cases, the variable $\eta$ increases with the Subcases a, b, c, and possibly, d. Now, it appears that $\varphi^{\eta \lambda}$ nondecreases from a subcase to its sequel. It is also non decreasing according to $\eta$ within each subcase (since $p_{x}^{\prime-1}$ is increasing). At the same time, the variables $\alpha_{\min }^{\eta \lambda}$ and $\alpha_{\max }^{\eta \lambda}$ do not increase from a subcase to its sequel, and within each subcase (since $p_{x}^{\prime}<0$ ).

In Case III, $\varphi^{\eta \lambda}$ is non decreasing because $p_{x}^{-1}$ is decreasing. Again, $\alpha_{\min }^{\eta \lambda}$ and $\alpha_{\max }^{\eta \lambda}$ do not increase because $p_{x}^{\prime}<0$.

At last, $\varphi^{\eta \lambda}$ is non decreasing according to $\eta$, while $\alpha_{\min }^{\eta \lambda}$ and $\alpha_{\max }^{\eta \lambda}$ are non increasing according to $\eta$.

Variation according to $\lambda$. Notice that $\lambda$ decreases with the Cases I, II, III, IV, and V. It also appears that $\varphi^{\eta \lambda}, \alpha_{\min }^{\eta \lambda}$, and $\alpha_{\max }^{\eta \lambda}$ do not decrease from a case to its sequel (the subcases have to be put together properly). Now, within the Cases I, II, IV, and $\mathrm{V}, \varphi^{\eta \lambda}, \alpha_{\min }^{\eta \lambda}$, and $\alpha_{\max }^{\eta \lambda}$ are constant according to $\lambda$. Within the Cases III, $\varphi^{\eta \lambda}, \alpha_{\min }^{\eta \lambda}$, and $\alpha_{\max }^{\eta \lambda}$ are non increasing according to $\lambda$ (because $\lambda \mapsto \frac{\eta}{p_{x}^{\prime}\left(p_{x}^{-1}(\lambda)\right)}$ and $\lambda \mapsto p_{x}^{-1}(\lambda)$ decrease).

At last, $\varphi^{\eta \lambda}, \alpha_{\min }^{\eta \lambda}$, and $\alpha_{\max }^{\eta \lambda}$ are non increasing according to $\lambda$.

Now, in order to prove (12), it is still necessary to verify $\lambda_{1}<\lambda_{2} \Rightarrow$ $\alpha_{\min }^{\eta \lambda_{1}} \geq \alpha_{\max }^{\eta \lambda_{2}}$. It is noticed that $\alpha_{\min }^{\eta \lambda} \neq \alpha_{\max }^{\eta \lambda}$ is possible only in the Cases IIa, $\mathrm{IIb}, \mathrm{IV} \mathrm{c}$, and IVd. These are the only cases that pose a slight difficulty. Now, it happens that the property holds at the interfaces I/IIa, IIa/III, I/IIb, IIb/III, $\mathrm{III} / \mathrm{IV} \mathrm{c}, \mathrm{III} / \mathrm{IV} \mathrm{c}, \mathrm{IVd} / \mathrm{V}$, and IVd/V, which end the proof.

For example, let us consider in detail the interface IIb/III. In particular, let $\lambda_{1}, \lambda_{2}, \eta$ be such that,

$$
\left\{\begin{array}{l}
\varphi_{1}(x)<p_{x}^{-1}\left(\lambda_{1}\right)<\varphi_{2}(x) \quad \text { [Case III]. } \\
p_{x}^{-1}\left(\lambda_{2}\right)=\varphi_{1}(x) \text { and } \alpha_{1}(x) \leq \frac{\eta}{p_{x}^{\prime}\left(\varphi_{1}(x)\right)} \leq \alpha_{2}(x) \quad \text { [Case IIb] }
\end{array}\right.
$$

(It is noteworthy that $\lambda_{1}<\lambda_{2}$ since $p_{x}^{-1}$ decreases.)

Then, $\varphi_{L}=\varphi_{1}(x)$ and $\varphi_{R}=\varphi_{2}(x)$ in III.

From III, it is then deduced $\varphi_{L} \leq p_{x}^{-1}\left(\lambda_{1}\right) \leq \varphi_{R}$ and $\alpha_{\min }^{\eta \lambda_{1}}(x)=$ $\alpha_{\max }^{\eta \lambda_{1}}(x)=\frac{\eta}{p_{x}^{\prime}\left(p_{x}^{-1}\left(\lambda_{1}\right)\right)}$.

Now, from II, it is derived $\alpha_{\max }^{\eta \lambda_{2}}(x)=\frac{\eta}{p_{x}^{\prime}\left(\varphi_{1}(x)\right)}$.

Since $\varphi_{1}(x) \leq p_{x}^{-1}\left(\lambda_{1}\right)$ and $f \mapsto \frac{\eta}{p_{x}^{\prime}(f)}$ increases, it is deduced $\alpha_{\max }^{\eta \lambda_{2}}(x) \leq \alpha_{\min }^{\eta \lambda_{1}}(x)$.

Thus, (12) holds for IIb/III.

Continuity. First, it is noticed that $\lambda, \eta \mapsto \varphi^{\eta \lambda}, \eta \mapsto \alpha_{\min }^{\eta \lambda}$, and $\eta \mapsto \alpha_{\max }^{\eta \lambda}$ are continuous according to the piecewise definition of Appendix A.

Now, there is more difficulty about $\lambda \mapsto \alpha^{\eta \lambda}$. When $\alpha_{\min }^{\eta \lambda}=\alpha_{\max }^{\eta \lambda}$, it is easy to check the contituity of $\lambda \mapsto \alpha_{\min }^{\eta \lambda}$ and $\lambda \mapsto \alpha_{\max }^{\eta \lambda}$. The problem comes from the Cases IIa, IIb, IVc, and IVd, for which could happen $\alpha_{\min }^{\eta \lambda} \neq \alpha_{\max }^{\eta \lambda}$. In order to recover (15), it is just sufficient to prove

$$
\alpha_{\min }^{\eta \lambda_{1}}(x) \underset{\lambda_{1} \rightarrow \lambda_{2}}{\stackrel{\lambda_{1}<\lambda_{2}}{\longrightarrow}} \alpha_{\max }^{\eta \lambda_{2}}(x) \quad \text { and } \quad \alpha_{\max }^{\eta \lambda_{1}}(x) \stackrel{\lambda_{1}>\lambda_{2}}{\lambda_{1} \rightarrow \lambda_{2}} \alpha_{\min }^{\eta \lambda_{2}}(x),
$$

at the interfaces I/IIa, IIa/III, I/IIb, IIb/III, III/IVc, III/IVc, IVd/V, and IVd/V, and that these convergences are locally uniform with $\eta$. These verifications are left to the reader. However, we will consider in detail the interface IIb/III.

Let $\lambda_{1}, \lambda_{2}, \eta$ be such that

$$
\left\{\begin{array}{l}
p_{x}^{-1}\left(\lambda_{2}\right)=\varphi_{1}(x) \text { and } \alpha_{1}(x) \leq \frac{\eta}{p_{x}^{\prime}\left(\varphi_{1}(x)\right)} \leq \alpha_{2}(x) \quad \text { [Case IIb] } \\
\varphi_{1}(x)<p_{x}^{-1}\left(\lambda_{1}\right)<\varphi_{2}(x) \quad \text { [case III]. }
\end{array}\right.
$$

(In this case, it is noteworthy that $\eta$ belongs to a compact set.)

Then, $\varphi_{L}=\varphi_{1}(x)$ and $\varphi_{R}=\varphi_{2}(x)$ in III.

From III, it is then deduced $\varphi_{L} \leq p_{x}^{-1}\left(\lambda_{1}\right) \leq \varphi_{R}$ and $\alpha_{\min }^{\eta \lambda_{1}}(x)=$ $\alpha_{\max }^{\eta \lambda_{1}}(x)=\frac{\eta}{p_{x}^{\prime}\left(p_{x}^{-1}\left(\lambda_{1}\right)\right)}$. 
Now, from II, it is derived $\alpha_{\max }^{\eta \lambda_{2}}(x)=\frac{\eta}{p_{x}^{\prime}\left(\varphi_{1}(x)\right)}$.

Since $\lim _{\lambda_{1} \rightarrow \lambda_{2}} p_{x}^{-1}\left(\lambda_{1}\right)=\varphi_{1}(x), f \stackrel{\eta}{f} \frac{\eta}{p_{x}^{\prime}(f)}$ is continuous, and $\eta$ belongs to a compact subset of $\mathbb{R}^{-*}, \alpha_{\min }^{\eta p_{x}^{\prime}(x) \frac{\lambda_{1}<\lambda_{2}}{\lambda_{1} \rightarrow \lambda_{2}}} \alpha_{\max }^{\eta \lambda_{2}}(x)$
uniformly with $\eta$.

Thus, (15) holds for IIb/III.

\section{REFERENCES}

[1] S.J. Benkovski, M.G. Monticino, and J.R. Weisinger, A survey of the Search Theory literature, Nav Res Logist 38 (1991), 469-491.

[2] S.S. Brown, Optimal search for a moving target in discrete time and space, Oper Res 28 (1980), 1275-1289.

[3] F. Dambreville and J.P. Le Cadre, "Spatial and temporal optimization of search efforts for the detection of a Markovian target," Presented at the 16th IMACS World Congress, Lausanne, Switzerland, 2000.

[4] J. de Guenin, Optimum distribution of effort: An extension of the Koopman Basic Theory, Oper Res 9 (1961), 1-7.

[5] V.F. Dem'yanov and V.N. Malozemov, Introduction to Minimax, Dover, New York, 1990.

[6] A. Garnaev, "Search games and other applications of game theory," Lecture notes in economics and mathematical systems, Volume 485, Springer, Berlin, 2000.

[7] K. Iida, R. Hohzaki, and K. Sato, Hide-and-search game with the risk criterion, J Oper Res Soc Jpn 37 (1994), 287-296.
[8] B.O. Koopman, Search and screening: General principle with historical applications, MORS Heritage Series, Alexandria, VA, 1999.

[9] T. Nakai, Search models with continuous effort under various criteria, J Oper Res Soc Jpn 31 (1988), 335-351.

[10] U. Pursiheimo, On the optimal search for a target whose motion is conditionally deterministic with stochastic initial conditions on location and parameters, SIAM J Appl Math 22(1) (1977), 105-114.

[11] U. Pursiheimo, Conjugate duality in the optimization of the search for a target with generalized conditionally determinitic motion, J Optim Theory Appl 3 (1980), 261-275.

[12] M. Sakaguchi, Two-sided search games, J Oper Res Soc Jpn 16 (1973), 207-225.

[13] M. Sakaguchi, A two-sided resource allocation game in search for a stationary object, Math Japonica 32 (1987), 979991.

[14] L.D. Stone, Theory of optimal search, 2nd ed., Operations Research Society of America, Arlington, VA, 1989.

[15] W.R. Stromquist and L.D. Stone, Constrained optimization of functionals with Search Theory applications, Math Oper Res 6 (1981), 518-529.

[16] D.H. Wagner, W.C. Mylander, and T.J. Sanders (Editors), Naval operations analysis, Chapter 5, 3rd ed., Naval Institute Press, Annapolis, MD, 1999.

[17] A.R. Washburn, Search for a moving Target: The FAB algorithm, Oper Res 31 (1983), 739-751. 